\title{
STRUCTURE OF SIMPLE MULTIPLICATIVE HOM-JORDAN ALGEBRAS
}

\author{
CHENRUI YAO, YAO MA, AND LIANGYUN CHEN
}

\begin{abstract}
We study the structure of simple multiplicative Hom-Jordan algebras. We discuss equivalent conditions for multiplicative Hom-Jordan algebras to be solvable, simple, and semi-simple. Moreover, we give a theorem on the classification of simple multiplicative Hom-Jordan algebras and obtain some propositions about bimodules of multiplicative Hom-Jordan algebras.
\end{abstract}

\section{INTRODUCTION}

Algebras where the identities defining the structure are twisted by a homomorphism are called Hom-algebras. These algebras have recently been investigated by many authors. The theory of Hom-algebras started from Hom-Lie algebras introduced and discussed in [6, 10, 11, 12. Hom-associative algebras were introduced in [15], while Hom-Jordan algebras were introduced in [14] as twisted generalization of Jordan algebras.

In recent years, vertex operator algebras are becoming more and more popular because of their importance. In [9], by using the structure of Heisenberg algebras, Lam constructed a vertex operator algebra such that the weight two space $V_{2} \cong J$ for a given simple Jordan algebra $J$ of type A, B or $\mathrm{C}$ over $\mathbb{C}$. In [2, Ashihara gave a counterexample to the following assertion: If $R$ is a subalgebra of the Griess algebra, then the weight two space of the vertex operator subalgebra $\operatorname{VOA}(R)$ generated by $R$ coincides with $R$ by using a vertex operator algebra associated with the simple Jordan algebra of type D. H. B. Zhao constructed simple quotients $\bar{V}_{\mathscr{J}, r}$ for $r \in \mathbb{Z}_{\neq 0}$ using dual-pair type constructions, where $\bar{V}_{\mathscr{J}, r}$ is such that $\left(\bar{V}_{\mathscr{J}, r}\right)_{0}=\mathbb{C} 1,\left(\bar{V}_{\mathscr{J}, r}\right)_{1}=\{0\}$, and $\left(\bar{V}_{\mathscr{J}, r}\right)_{2}$ is isomorphic to the type B Jordan algebra $\mathscr{J}$. Moreover, in his paper [19] he reproved that $V_{\mathscr{J}, r}$ is simple if $r \notin \mathbb{Z}$.

The structure of Hom-algebras seems to be more complex because of the variety of twisted maps. But the structure of the original algebras is pretty clear. So one of the ways to study the structure of Hom-algebras is to look for relationships between Hom-algebras and their induced algebras. In [15], Makhlouf and Silvestrov

2020 Mathematics Subject Classification. 17C10, 17C20, 17C50, 17B10, 17A30.

Supported by NNSF of China (Nos. 11771069 and 11801066), NSF of Jilin province (No. 20170101048JC), the project of Jilin Province Department of Education (No. JJKH20180005K), and the Fundamental Research Funds for the Central Universities (No. 130014801). 
introduced the structure of Hom-associative algebras and Hom-Leibniz algebras together with their induced algebras. In [13], X. X. Li studied the structure of multiplicative Hom-Lie algebras and gave equivalent conditions for a multiplicative Hom-Lie algebra to be solvable, simple, and semi-simple. By a similar analysis, in this paper we successfully generalize the above results to Hom-Jordan algebras.

It is well known that simple algebras play an important role in structure theory. Similarly, it is necessary to study simple Hom-algebras in the theory of Homalgebras. In [5], X. Chen and W. Han gave a classification theorem about simple multiplicative Hom-Lie algebras. Using some theorems obtained in Section 3 , we generalize the above theorem to Hom-Jordan algebras.

Nowadays, one of the current trends in mathematics has to do with representations and deformations. The two topics are important tools in most parts of mathematics and physics. Representations of Hom-Lie algebras were introduced and studied in [4, 17], but representations of Hom-Jordan algebras came much later. In 2018, Attan gave the definition of bimodules of Hom-Jordan algebras in his paper [3]. In [1], Agrebaoui, Benali, and Makhlouf studied representations of simple Hom-Lie algebras and gave some propositions about them. In this paper, we also give some propositions about bimodules of Hom-Jordan algebras using similar methods.

The paper is organised as follows: In Section 2, we introduce some basic definitions and prove a few lemmas which can be used in what follows. In Section 3, we mainly show three important results, Theorems 3.3, 3.5. and 3.6. which are about solvability, simplicity, and semi-simplicity of multiplicative Hom-Jordan algebras, respectively. In Section 4 we first give a result, Theorem 4.1 . on the construction of $n$-dimensional simple Hom-Jordan algebras. Next we give our main theorem in this section, Theorem 4.3. which is about classification of simple multiplicative HomJordan algebras. In Section 5, we prove a very important result, Theorem 5.5, which is about relationships between bimodules of Hom-Jordan algebras and modules of their induced Jordan algebras. Moreover, some propositions about bimodules of simple Hom-Jordan algebras are also obtained as an application of Theorem 5.5

\section{Preliminaries}

Definition 2.1 ([16, pp. 152-157]). A Jordan algebra $J$ over a field $\mathrm{F}$ is an algebra satisfying, for any $x, y \in J$,

(1) $x \circ y=y \circ x$

(2) $\left(x^{2} \circ y\right) \circ x=x^{2} \circ(y \circ x)$.

Definition 2.2 ([14]). A Hom-Jordan algebra over a field $\mathrm{F}$ is a triple $(V, \mu, \alpha)$ consisting of a linear space $V$, a bilinear map $\mu: V \times V \rightarrow V$ which is commutative, and a linear map $\alpha: V \rightarrow V$ satisfying, for any $x, y \in V$,

$$
\mu\left(\alpha^{2}(x), \mu(y, \mu(x, x))\right)=\mu(\mu(\alpha(x), y), \alpha(\mu(x, x))),
$$

where $\alpha^{2}=\alpha \circ \alpha$.

Definition 2.3. A Hom-Jordan algebra $(V, \mu, \alpha)$ is called multiplicative if for any $x, y \in V, \alpha(\mu(x, y))=\mu(\alpha(x), \alpha(y))$. 
Definition 2.4 ([7]). A subspace $W \subseteq V$ is a Hom-subalgebra of $(V, \mu, \alpha)$ if $\alpha(W) \subseteq W$ and

$$
\mu(x, y) \in W, \quad \forall x, y \in W .
$$

Definition 2.5 ([7]). A subspace $W \subseteq V$ is a Hom-ideal of $(V, \mu, \alpha)$ if $\alpha(W) \subseteq W$ and

$$
\mu(x, y) \in W, \quad \forall x \in W, y \in V .
$$

Definition 2.6 ([7]). Let $(V, \mu, \alpha)$ and $\left(V^{\prime}, \mu^{\prime}, \beta\right)$ be two Hom-Jordan algebras. A linear map $\phi: V \rightarrow V^{\prime}$ is said to be a homomorphism of Hom-Jordan algebras if

(1) $\phi(\mu(x, y))=\mu^{\prime}(\phi(x), \phi(y))$;

(2) $\phi \circ \alpha=\beta \circ \phi$.

In particular, $\phi$ is an isomorphism if $\phi$ is bijective.

Definition 2.7. A Hom-Jordan algebra $(V, \mu, \alpha)$ is called a Jordan-type HomJordan algebra if there exists a Jordan algebra $\left(V, \mu^{\prime}\right)$ such that

$$
\mu(x, y)=\alpha\left(\mu^{\prime}(x, y)\right)=\mu^{\prime}(\alpha(x), \alpha(y)), \quad \forall x, y \in V,
$$

and $\left(V, \mu^{\prime}\right)$ is called the induced Jordan algebra.

Lemma 2.8. (1) Suppose that $(V, \mu)$ is a Jordan algebra and $\alpha: V \rightarrow V$ is a homomorphism. Then $(V, \tilde{\mu}, \alpha)$ is a multiplicative Hom-Jordan algebra with $\tilde{\mu}(x, y)=\alpha(\mu(x, y)), \forall x, y \in V$.

(2) Suppose that $(V, \mu, \alpha)$ is a multiplicative Hom-Jordan algebra and $\alpha$ is invertible. Then $(V, \mu, \alpha)$ is a Jordan-type Hom-Jordan algebra and its induced Jordan algebra is $\left(V, \mu^{\prime}\right)$ with $\mu^{\prime}(x, y)=\alpha^{-1}(\mu(x, y)), \forall x, y \in V$.

Proof. (1). We have that $\tilde{\mu}$ is commutative, since $\mu$ is commutative.

For all $x, y \in V$, we have

$$
\begin{aligned}
& \tilde{\mu}\left(\alpha^{2}(x), \tilde{\mu}(y, \tilde{\mu}(x, x))\right)=\alpha\left(\mu\left(\alpha^{2}(x), \alpha(\mu(y, \alpha(\mu(x, x))))\right)\right) \\
& \quad=\mu\left(\alpha^{3}(x), \mu\left(\alpha^{2}(y), \mu\left(\alpha^{3}(x), \alpha^{3}(x)\right)\right)\right)=\mu\left(\mu\left(\alpha^{3}(x), \alpha^{2}(y)\right), \mu\left(\alpha^{3}(x), \alpha^{3}(x)\right)\right) \\
& \quad=\alpha\left(\mu\left(\alpha(\mu(\alpha(x), y)), \alpha^{2}(\mu(x, x))\right)\right)=\tilde{\mu}(\tilde{\mu}(\alpha(x), y), \alpha(\tilde{\mu}(x, x))),
\end{aligned}
$$

which implies that $(V, \tilde{\mu}, \alpha)$ is a Hom-Jordan algebra. We see that

$$
\alpha(\tilde{\mu}(x, y))=\alpha^{2}(\mu(x, y))=\alpha(\mu(\alpha(x), \alpha(y)))=\tilde{\mu}(\alpha(x), \alpha(y)),
$$

which implies that $(V, \tilde{\mu}, \alpha)$ is multiplicative. Hence, $(V, \tilde{\mu}, \alpha)$ is a multiplicative Hom-Jordan algebra.

(2). We have that $\mu^{\prime}$ is commutative, since $\mu$ is commutative.

For any $x, y \in V$, we have

$$
\begin{aligned}
& \mu^{\prime}\left(\mu^{\prime}\left(\mu^{\prime}(x, x), y\right), x\right)=\alpha^{-1}\left(\mu\left(\alpha^{-1}\left(\mu\left(\alpha^{-1}(\mu(x, x)), y\right)\right), x\right)\right) \\
& \quad=\alpha^{-3}\left(\mu\left(\mu(\mu(x, x), \alpha(y)), \alpha^{2}(x)\right)\right)=\alpha^{-3}(\mu(\alpha(\mu(x, x)), \mu(\alpha(y), \alpha(x)))) \\
& \quad=\alpha^{-1}\left(\mu\left(\alpha^{-1}(\mu(x, x)), \alpha^{-1}(\mu(y, x))\right)\right)=\mu^{\prime}\left(\mu^{\prime}(x, x), \mu^{\prime}(y, x)\right),
\end{aligned}
$$

which implies that $\left(V, \mu^{\prime}\right)$ is a Jordan algebra.

It is obvious that $\mu(x, y)=\alpha\left(\mu^{\prime}(x, y)\right)=\mu^{\prime}(\alpha(x), \alpha(y))$ for any $x, y \in V$. Hence, $(V, \mu, \alpha)$ is a Jordan-type Hom-Jordan algebra. 
Definition 2.9. Suppose that $(V, \mu, \alpha)$ is a Hom-Jordan algebra. Define its derived sequence as follows:

$$
V^{(1)}=\mu(V, V), \quad V^{(2)}=\mu\left(V^{(1)}, V^{(1)}\right), \ldots, V^{(k)}=\mu\left(V^{(k-1)}, V^{(k-1)}\right), \ldots
$$

If there exists $m \in \mathbb{Z}^{+}$such that $V^{(m)}=0$, then $(V, \mu, \alpha)$ is called solvable.

Definition 2.10. Suppose that $(V, \mu, \alpha)$ is a Hom-Jordan algebra and $\alpha \neq 0$. If $(V, \mu, \alpha)$ has no nontrivial Hom-ideals and satisfies $\mu(V, V)=V$, then $(V, \mu, \alpha)$ is called simple. If

$$
V=V_{1} \oplus V_{2} \oplus \cdots \oplus V_{s}
$$

where $V_{i}(1 \leq i \leq s)$ are simple Hom-ideals of $(V, \mu, \alpha)$, then $(V, \mu, \alpha)$ is called semi-simple.

Proposition 2.11. Suppose that $\left(V_{1}, \tilde{\mu_{1}}, \alpha\right)$ and $\left(V_{2}, \tilde{\mu_{2}}, \beta\right)$ are two Jordan-type Hom-Jordan algebras and $\beta$ is injective. Then $\phi$ is an isomorphism from $\left(V_{1}, \tilde{\mu_{1}}, \alpha\right)$ to $\left(V_{2}, \tilde{\mu_{2}}, \beta\right)$ if and only if $\phi$ is an isomorphism between their induced Jordan algebras $\left(V_{1}, \mu_{1}\right)$ and $\left(V_{2}, \mu_{2}\right)$ and $\phi$ satisfies $\beta \circ \phi=\phi \circ \alpha$.

Proof. $(\Rightarrow)$ For any $x, y \in V_{1}$, we have

$$
\phi\left(\tilde{\mu_{1}}(x, y)\right)=\tilde{\mu_{2}}(\phi(x), \phi(y))
$$

i.e.,

$$
\phi\left(\alpha\left(\mu_{1}(x, y)\right)\right)=\beta\left(\mu_{2}(\phi(x), \phi(y))\right) .
$$

Note that $\phi \circ \alpha=\beta \circ \phi$. We have

$$
\beta\left(\phi\left(\mu_{1}(x, y)\right)\right)=\beta\left(\mu_{2}(\phi(x), \phi(y))\right) .
$$

Since $\beta$ is injective, we have

$$
\phi\left(\mu_{1}(x, y)\right)=\mu_{2}(\phi(x), \phi(y)),
$$

which implies that $\phi$ is an isomorphism from $\left(V_{1}, \mu_{1}\right)$ to $\left(V_{2}, \mu_{2}\right)$.

$(\Leftarrow)$ For any $x, y \in V_{1}$, we have

$$
\begin{aligned}
\phi\left(\tilde{\mu_{1}}(x, y)\right) & =\phi\left(\alpha\left(\mu_{1}(x, y)\right)\right)=\beta\left(\phi\left(\mu_{1}(x, y)\right)\right) \\
& =\beta\left(\mu_{2}(\phi(x), \phi(y))\right)=\tilde{\mu_{2}}(\phi(x), \phi(y)) .
\end{aligned}
$$

Note that $\beta \circ \phi=\phi \circ \alpha$. We have that $\phi$ is an isomorphism from $\left(V_{1}, \tilde{\mu_{1}}, \alpha\right)$ to $\left(V_{2}, \tilde{\mu_{2}}, \beta\right)$.

Lemma 2.12. Simple multiplicative Hom-Jordan algebras with $\alpha \neq 0$ are Jordantype Hom-Jordan algebras.

Proof. Suppose that $(V, \mu, \alpha)$ is a simple multiplicative Hom-Jordan algebra. According to Lemma 2.8 (2), we only need to show that $\alpha$ is invertible. If $\alpha$ is not invertible, then $\operatorname{Ker}(\alpha) \neq 0$. It is obvious that $\alpha(\operatorname{Ker}(\alpha)) \subseteq \operatorname{Ker}(\alpha)$. For any $x \in \operatorname{Ker}(\alpha), y \in V$, we have

$$
\alpha(\mu(x, y))=\mu(\alpha(x), \alpha(y))=\mu(0, \alpha(y))=0,
$$

which implies that $\mu(\operatorname{Ker}(\alpha), V) \subseteq \operatorname{Ker}(\alpha)$. Then $\operatorname{Ker}(\alpha)$ is a nontrivial Homideal of $(V, \mu, \alpha)$, contradicting the assumption that $(V, \mu, \alpha)$ is simple. Therefore, 
$\operatorname{Ker}(\alpha)=0$, i.e., $\alpha$ is invertible. Hence, $(V, \mu, \alpha)$ is a Jordan-type Hom-Jordan algebra.

Now we give a corollary of Proposition 2.11 using Lemma 2.12

Corollary 2.13. Two simple multiplicative Hom-Jordan algebras $\left(V_{1}, \tilde{\mu}_{1}, \alpha\right)$ and $\left(V_{2}, \tilde{\mu}_{2}, \beta\right)$ are isomorphic if and only if there exists an isomorphism $\phi$ between their induced Jordan algebras $\left(V_{1}, \mu_{1}\right)$ and $\left(V_{2}, \mu_{2}\right)$ and $\phi$ satisfies $\beta \circ \phi=\phi \circ \alpha$.

\section{Structure of multiplicative Hom-Jordan algebras}

In this section, we discuss sufficient and necessary conditions for multiplicative Hom-Jordan algebras to be solvable, simple, and semi-simple.

Proposition 3.1. Suppose that $(V, \mu, \alpha)$ is a multiplicative Hom-Jordan algebra and $I$ is a Hom-ideal of $(V, \mu, \alpha)$. Then $(V / I, \bar{\mu}, \bar{\alpha})$ is a multiplicative Hom-Jordan algebra, where $\bar{\mu}(\bar{x}, \bar{y})=\overline{\mu(x, y)}, \bar{\alpha}(\bar{x})=\overline{\alpha(x)}$ for all $\bar{x}, \bar{y} \in V / I$.

Proof. We have that $\bar{\mu}$ is commutative, since $\mu$ is commutative.

For any $\bar{x}, \bar{y} \in V / I$, we have

$$
\begin{aligned}
\bar{\mu}\left(\bar{\alpha}^{2}(\bar{x}), \bar{\mu}(\bar{y}, \bar{\mu}(\bar{x}, \bar{x}))\right) & =\overline{\mu\left(\alpha^{2}(x), \mu(y, \mu(x, x))\right)} \\
& =\overline{\mu(\mu(\alpha(x), y), \alpha(\mu(x, x)))} \\
& =\bar{\mu}(\bar{\mu}(\bar{\alpha}(\bar{x}), \bar{y}), \bar{\alpha}(\bar{\mu}(\bar{x}, \bar{x}))) .
\end{aligned}
$$

Hence, $(V / I, \bar{\mu}, \bar{\alpha})$ is a Hom-Jordan algebra. Moreover, we see that

$$
\bar{\alpha}(\bar{\mu}(\bar{x}, \bar{y}))=\overline{\alpha(\mu(x, y))}=\overline{\mu(\alpha(x), \alpha(y))}=\bar{\mu}(\bar{\alpha}(\bar{x}, \bar{y})),
$$

which implies that $(V / I, \bar{\mu}, \bar{\alpha})$ is multiplicative.

Corollary 3.2. Suppose that $(V, \mu, \alpha)$ is a multiplicative Hom-Jordan algebra and satisfies $\alpha^{2}=\alpha$. Then $(V / \operatorname{Ker}(\alpha), \bar{\mu}, \bar{\alpha})$ is a Jordan-type Hom-Jordan algebra.

Proof. If $\alpha$ is invertible, $\operatorname{Ker}(\alpha)=0$. According to Lemma 2.8 (2), the conclusion is valid. If $\alpha$ isn't invertible, according to the proof of Lemma 2.12, we have that $\operatorname{Ker}(\alpha)$ is a Hom-ideal of $(V, \mu, \alpha)$. Then we have that $(V / \operatorname{Ker}(\alpha), \bar{\mu}, \bar{\alpha})$ is a multiplicative Hom-Jordan algebra according to Proposition 3.1. Now we show that $\bar{\alpha}$ is invertible on $V / \operatorname{Ker}(\alpha)$.

Assume that $\bar{x} \in \operatorname{Ker}(\bar{\alpha})$. Then we have $\overline{\alpha(x)}=\bar{\alpha}(\bar{x})=\overline{0}$, i.e., $\alpha(x) \in \operatorname{Ker}(\alpha)$. Note that $\alpha^{2}=\alpha$. We have

$$
\alpha(x)=\alpha^{2}(x)=\alpha(\alpha(x))=0,
$$

which implies that $x \in \operatorname{Ker}(\alpha)$, i.e., $\bar{x}=\overline{0}$. Hence, $\bar{\alpha}$ is invertible. According to Lemma $2.8(2),(V / \operatorname{Ker}(\alpha), \bar{\mu}, \bar{\alpha})$ is a Jordan-type Hom-Jordan algebra.

Theorem 3.3. Suppose that $(V, \mu, \alpha)$ is a multiplicative Hom-Jordan algebra and $\alpha$ is invertible. Then $(V, \mu, \alpha)$ is solvable if and only if its induced Jordan algebra $\left(V, \mu^{\prime}\right)$ is solvable. 
Proof. Denote the derived series of $\left(V, \mu^{\prime}\right)$ and $(V, \mu, \alpha)$ by $V^{(i)}, \tilde{V}^{(i)}(i=1,2, \ldots)$, respectively. Suppose that $\left(V, \mu^{\prime}\right)$ is solvable. Then there exists $m \in \mathbb{Z}^{+}$such that $V^{(m)}=0$. Note that

$$
\begin{gathered}
\tilde{V}^{(1)}=\mu(V, V)=\alpha\left(\mu^{\prime}(V, V)\right)=\alpha\left(V^{(1)}\right), \\
\tilde{V}^{(2)}=\mu\left(\tilde{V}^{(1)}, \tilde{V}^{(1)}\right)=\mu\left(\alpha\left(V^{(1)}\right), \alpha\left(V^{(1)}\right)\right)=\alpha^{2}\left(\mu^{\prime}\left(V^{(1)}, V^{(1)}\right)\right)=\alpha^{2}\left(V^{(2)}\right) .
\end{gathered}
$$

We have $\tilde{V}^{(m)}=\alpha^{m}\left(V^{(m)}\right)$ by induction. Hence, $\tilde{V}^{(m)}=0$, i.e., $(V, \mu, \alpha)$ is solvable.

On the other hand, assume that $(V, \mu, \alpha)$ is solvable. Then there exists $m \in \mathbb{Z}^{+}$ such that $\tilde{V}^{(m)}=0$. We have $\tilde{V}^{(m)}=\alpha^{m}\left(V^{(m)}\right)$ by the above proof. Hence we have $V^{(m)}=0$, since $\alpha$ is invertible. Therefore, $\left(V, \mu^{\prime}\right)$ is solvable.

Lemma 3.4. Suppose that an algebra $\mathcal{A}$ over $\mathrm{F}$ can be decomposed into the unique direct sum of simple ideals $\mathcal{A}=\bigoplus_{i=1}^{s} \mathcal{A}_{i}$, where $\mathcal{A}_{i}$ aren't isomorphic to each other and $\alpha \in \operatorname{Aut}(\mathcal{A})$. Then $\alpha\left(\mathcal{A}_{i}\right)=\mathcal{A}_{i}(1 \leq i \leq s)$.

Proof. For any $1 \leq i \leq s$, we have

$$
\alpha\left(\mathcal{A}_{i}\right) \mathcal{A}=\alpha\left(\mathcal{A}_{i}\right) \alpha(\mathcal{A})=\alpha\left(\mathcal{A}_{i} \mathcal{A}\right) \subseteq \alpha\left(\mathcal{A}_{i}\right)
$$

since $\mathcal{A}_{i}$ are ideals of $\mathcal{A}$. Similarly, we have $\mathcal{A} \alpha\left(\mathcal{A}_{i}\right) \subseteq \alpha\left(\mathcal{A}_{i}\right)$. Hence, $\alpha\left(\mathcal{A}_{i}\right)$ are also ideals of $\mathcal{A}$. Moreover, $\alpha\left(\mathcal{A}_{i}\right)$ are simple since $\mathcal{A}_{i}$ are simple.

Note that $\mathcal{A}=\bigoplus_{i=1}^{s} \mathcal{A}_{i}$. We have

$$
\mathcal{A}=\alpha(\mathcal{A})=\alpha\left(\oplus_{i=1}^{s} \mathcal{A}_{i}\right)=\oplus_{i=1}^{s} \alpha\left(\mathcal{A}_{i}\right) .
$$

Note that the decomposition is unique. There exists $1 \leq j \leq s$ such that $\alpha\left(\mathcal{A}_{i}\right)=\mathcal{A}_{j}$ for any $1 \leq i \leq s$.

If $j \neq i$, then we have

$$
\mathcal{A}_{i} \cong \alpha\left(\mathcal{A}_{i}\right)=\mathcal{A}_{j},
$$

contradicting the assumption that $\mathcal{A}_{i}$ aren't isomorphic to each other. Hence, we have $\alpha\left(\mathcal{A}_{i}\right)=\mathcal{A}_{i}(1 \leq i \leq s)$ for any $s \in \mathbb{N}$.

Theorem 3.5. (1) Suppose that $(V, \mu, \alpha)$ is a simple multiplicative Hom-Jordan algebra. Then its induced Jordan algebra $\left(V, \mu^{\prime}\right)$ is semi-simple. Moreover, $\left(V, \mu^{\prime}\right)$ can be decomposed into a direct sum of isomorphic simple ideals; in addition, $\alpha$ acts simply transitively on simple ideals of the induced Jordan algebra.

(2) Suppose that $\left(V, \mu^{\prime}\right)$ is a simple Jordan algebra and $\alpha \in \operatorname{Aut}(V)$. Define $\mu$ : $V \times V \rightarrow V$ by

$$
\mu(x, y)=\alpha\left(\mu^{\prime}(x, y)\right), \quad \forall x, y \in V .
$$

Then $(V, \mu, \alpha)$ is a simple multiplicative Hom-Jordan algebra.

Proof. (1) According to the proof of Lemma 2.8 (2) and Lemma 2.12 $\alpha$ is an automorphism both on $(V, \mu, \alpha)$ and $\left(V, \mu^{\prime}\right)$.

Suppose that $V_{1}$ is the maximal solvable ideal of $\left(V, \mu^{\prime}\right)$. Then there exists $m \in \mathbb{Z}^{+}$such that $V_{1}^{(m)}=0$. 
Note that

$$
\begin{gathered}
\mu^{\prime}\left(\alpha\left(V_{1}\right), V\right)=\mu^{\prime}\left(\alpha\left(V_{1}\right), \alpha(V)\right)=\alpha\left(\mu^{\prime}\left(V_{1}, V\right)\right) \subseteq \alpha\left(V_{1}\right), \\
\left(\alpha\left(V_{1}\right)\right)^{(m)}=\alpha\left(V_{1}^{(m)}\right)=0 .
\end{gathered}
$$

We have $\alpha\left(V_{1}\right)$ is also a solvable ideal of $\left(V, \mu^{\prime}\right)$. Then we have $\alpha\left(V_{1}\right) \subseteq V_{1}$. Moreover,

$$
\mu\left(V_{1}, V\right)=\alpha\left(\mu^{\prime}\left(V_{1}, V\right)\right) \subseteq \alpha\left(V_{1}\right) \subseteq V_{1},
$$

so $V_{1}$ is a Hom-ideal of $(V, \mu, \alpha)$. Then we have $V_{1}=0$ or $V_{1}=V$ since $(V, \mu, \alpha)$ is simple. If $V_{1}=V$, according to the proof of Theorem 3.3, we have

$$
\tilde{V}^{(m)}=\alpha^{m}\left(V^{(m)}\right)=\alpha^{m}\left(V_{1}^{(m)}\right)=0 ;
$$

on the other hand, $V=\mu(V, V)$ since $(V, \mu, \alpha)$ is simple. Then we have $\tilde{V}^{(m)}=V$, a contradiction. Hence, $V_{1}=0$. Therefore, $\left(V, \mu^{\prime}\right)$ is semi-simple.

Since $\left(V, \mu^{\prime}\right)$ is semi-simple, we have $V=\bigoplus_{i=1}^{s} V_{i}$, where $V_{i}(1 \leq i \leq s)$ are simple ideals of $\left(V, \mu^{\prime}\right)$. Because there may be isomorphic Jordan algebras in $V_{1}, V_{2}, \ldots, V_{s}$, we rearrange the order as follows:

$V=V_{11} \oplus V_{12} \oplus \cdots \oplus V_{1 m_{1}} \oplus V_{21} \oplus V_{22} \oplus \cdots \oplus V_{2 m_{2}} \oplus \cdots \oplus V_{t 1} \oplus V_{t 2} \oplus \cdots \oplus V_{t m_{t}}$, where

$$
\left(V_{i j}, \mu^{\prime}\right) \cong\left(V_{i k}, \mu^{\prime}\right), \quad 1 \leq j, k \leq m_{i}, i=1,2, \ldots, t .
$$

According to Lemma 3.4 we have

$$
\begin{gathered}
\alpha\left(V_{i 1} \oplus V_{i 2} \oplus \cdots \oplus V_{i m_{i}}\right)=V_{i 1} \oplus V_{i 2} \oplus \cdots \oplus V_{i m_{i}} \\
\mu\left(V_{i 1} \oplus V_{i 2} \oplus \cdots \oplus V_{i m_{i}}, V\right)=\alpha\left(\mu^{\prime}\left(V_{i 1} \oplus V_{i 2} \oplus \cdots \oplus V_{i m_{i}}, V\right)\right) \\
\subseteq \alpha\left(V_{i 1} \oplus V_{i 2} \oplus \cdots \oplus V_{i m_{i}}\right)=V_{i 1} \oplus V_{i 2} \oplus \cdots \oplus V_{i m_{i}}
\end{gathered}
$$

and so we have that $V_{i 1} \oplus V_{i 2} \oplus \cdots \oplus V_{i m_{i}}$ are Hom-ideals of $(V, \mu, \alpha)$. Since $(V, \mu, \alpha)$ is simple, we have $V_{i 1} \oplus V_{i 2} \oplus \cdots \oplus V_{i m_{i}}=0$ or $V$. So all but one $V_{i 1} \oplus V_{i 2} \oplus \cdots \oplus V_{i m_{i}}$ must be 0 . Without loss of generality, we can assume that

$$
V=V_{11} \oplus V_{12} \oplus \cdots \oplus V_{1 m_{1}} .
$$

When $m_{1}=1,\left(V, \mu^{\prime}\right)$ is simple. When $m_{1}>1$, if

$$
\alpha\left(V_{1 p}\right)=V_{1 p} \quad\left(1 \leq p \leq m_{1}\right)
$$

then $V_{1 p}$ is a nontrivial ideal of $(V, \mu, \alpha)$, which contradicts the fact that $(V, \mu, \alpha)$ is simple. Hence,

$$
\alpha\left(V_{1 p}\right)=V_{1 l} \quad\left(1 \leq l \neq p \leq m_{1}\right) .
$$

In addition, it is easy to show that $V_{11} \oplus \alpha\left(V_{11}\right) \oplus \cdots \oplus \alpha^{m_{1}-1}\left(V_{11}\right)$ is a Hom-ideal of $(V, \mu, \alpha)$. Therefore,

$$
V=V_{11} \oplus \alpha\left(V_{11}\right) \oplus \cdots \oplus \alpha^{m_{1}-1}\left(V_{11}\right) .
$$

That is, $\alpha$ acts simply transitively on simple ideals of the induced Jordan algebra. 
(2) Suppose that $\left(V, \mu^{\prime}\right)$ is a simple Jordan algebra. According to Lemma 2.8 (1), we have that $(V, \mu, \alpha)$ is a multiplicative Hom-Jordan algebra. Suppose that $V_{1}$ is a nontrivial Hom-ideal of $(V, \mu, \alpha)$; then we have

$$
\mu^{\prime}\left(V_{1}, V\right)=\alpha^{-1}\left(\mu\left(V_{1}, V\right)\right) \subseteq \alpha^{-1}\left(V_{1}\right)=V_{1} .
$$

So $V_{1}$ is a nontrivial ideal of $\left(V, \mu^{\prime}\right)$, a contradiction. So $(V, \mu, \alpha)$ has no nontrivial ideal. If $\mu(V, V) \varsubsetneqq V$, then

$$
\mu^{\prime}(V, V)=\alpha^{-1}(\mu(V, V)) \varsubsetneqq \alpha^{-1}(V)=V,
$$

contradicting the assumption that $\left(V, \mu^{\prime}\right)$ is a simple Jordan algebra. Hence, $(V, \mu, \alpha)$ is simple.

Theorem 3.6. (1) Suppose that $(V, \mu, \alpha)$ is a semi-simple multiplicative HomJordan algebra. Then $(V, \mu, \alpha)$ is a Jordan-type Hom-Jordan algebra and its induced Jordan algebra $\left(V, \mu^{\prime}\right)$ is also semi-simple.

(2) Suppose that $\left(V, \mu^{\prime}\right)$ is a semi-simple Jordan algebra and has the decomposition $V=\bigoplus_{i=1}^{s} V_{i}$, where $V_{i}(1 \leq i \leq s)$ are simple ideals of $\left(V, \mu^{\prime}\right)$, and $\alpha \in \operatorname{Aut}(V)$ satisfies $\alpha\left(V_{i}\right)=V_{i}(1 \leq i \leq s)$. Then $(V, \mu, \alpha)$ is a semi-simple multiplicative Hom-Jordan algebra and has a unique decomposition.

Proof. (1) According to the assumption, $(V, \mu, \alpha)$ has the decomposition $V=$ $\bigoplus_{i=1}^{s} V_{i}$, where $V_{i}(1 \leq i \leq s)$ are simple Hom-ideals of $(V, \mu, \alpha)$. Then $\left(V_{i}, \mu,\left.\alpha\right|_{V_{i}}\right)$ $(1 \leq i \leq s)$ are simple Hom-Jordan algebras. According to the proof of Lemma 2.12 $\left.\alpha\right|_{V_{i}}(1 \leq i \leq s)$ are invertible. Therefore, $\alpha$ is invertible on $V$. According to Lemma $2.8(2),(V, \mu, \alpha)$ is a Jordan-type Hom-Jordan algebra and its induced Jordan algebra is $\left(V, \mu^{\prime}\right)$, where $\mu^{\prime}(x, y)=\alpha^{-1}(\mu(x, y))$ for all $x, y \in V$.

According to the proof of Theorem 3.5 (2), $V_{i}(i=1,2, \ldots, s)$ are ideals of $\left(V, \mu^{\prime}\right)$. Moreover, $\left(V_{i}, \mu^{\prime}\right)$ are induced Jordan algebras of simple Hom-Jordan algebras $\left(V_{i}, \mu,\left.\alpha\right|_{V_{i}}\right)$, respectively. According to Theorem 3.5 (1), $\left(V_{i}, \mu^{\prime}\right)$ are semisimple Jordan algebras and can be decomposed into a direct sum of isomorphic simple ideals $V_{i}=V_{i 1} \oplus V_{i 2} \oplus \cdots \oplus V_{i m_{i}}$. Therefore, $\left(V, \mu^{\prime}\right)$ is semi-simple and has the decomposition into a direct sum of simple ideals

$$
V=V_{11} \oplus V_{12} \oplus \cdots \oplus V_{1 m_{1}} \oplus V_{21} \oplus V_{22} \oplus \cdots \oplus V_{2 m_{2}} \oplus \cdots \oplus V_{s 1} \oplus V_{s 2} \oplus \cdots \oplus V_{s m_{s}} .
$$

(2) According to Lemma $2.8(1),(V, \mu, \alpha)$ is a multiplicative Hom-Jordan algebra. For all $1 \leq i \leq s$, we have

$$
\mu\left(V_{i}, V\right)=\alpha\left(\mu^{\prime}\left(V_{i}, V\right)\right) \subseteq \alpha\left(V_{i}\right)=V_{i} .
$$

Note that $\alpha\left(V_{i}\right)=V_{i}$. We have that $V_{i}$ are Hom-ideals of $(V, \mu, \alpha)$.

If there exists $V_{i 0} \varsubsetneqq V_{i}$ which is a nontrivial Hom-ideal of $\left(V_{i}, \mu,\left.\alpha\right|_{V_{i}}\right)$, then we have

$$
\mu\left(V_{i 0}, V\right)=\mu\left(V_{i 0}, V_{1} \oplus V_{2} \oplus \cdots \oplus V_{s}\right)=\mu\left(V_{i 0}, V_{i}\right) \subseteq V_{i 0},
$$

so $V_{i 0}$ is a nontrivial Hom-ideal of $(V, \mu, \alpha)$. According to the proof of Theorem $3.5(2), V_{i 0}$ is also a nontrivial ideal of $\left(V, \mu^{\prime}\right)$. Hence, $V_{i 0}$ is also a nontrivial ideal of $\left(V_{i}, \mu^{\prime}\right)$, a contradiction. Hence, $V_{i}(i=1,2, \ldots, s)$ are simple Hom-ideals of $(V, \mu, \alpha)$. Therefore, $(V, \mu, \alpha)$ is semi-simple and has a unique decomposition. 
Proposition 3.7. Suppose that $(V, \mu, \alpha)$ is a multiplicative Hom-Jordan algebra satisfying $\alpha^{2}=\alpha$ and $\mu(\operatorname{Im}(\alpha), V) \subseteq \operatorname{Im}(\alpha)$. Then $(V, \mu, \alpha)$ is isomorphic to the decomposition into a direct sum of Hom-Jordan algebras

$$
V \cong(V / \operatorname{Ker}(\alpha)) \oplus \operatorname{Ker}(\alpha) .
$$

Proof. Set $V_{1}=(V / \operatorname{Ker}(\alpha)) \oplus \operatorname{Ker}(\alpha)$. According to Corollary 3.2 . $(V / \operatorname{Ker}(\alpha), \bar{\mu}, \bar{\alpha})$ is a Hom-Jordan algebra. It is obvious that $\left(\operatorname{Ker}(\alpha), \mu,\left.\alpha\right|_{\operatorname{Ker}(\alpha)}\right)$ is a Hom-Jordan algebra. Define $\mu_{1}: V_{1} \times V_{1} \rightarrow V_{1}$ and $\alpha_{1}: V_{1} \rightarrow V_{1}$ by

$$
\begin{aligned}
\mu_{1}\left(\left(\bar{x}, k_{1}\right),\left(\bar{y}, k_{2}\right)\right) & =\left(\overline{\mu(x, y)}, \mu\left(k_{1}, k_{2}\right)\right), \\
\alpha_{1}\left(\left(\bar{x}, k_{1}\right)\right) & =(\overline{\alpha(x)}, 0) .
\end{aligned}
$$

Then $\left(V_{1}, \mu_{1}, \alpha_{1}\right)$ is a Hom-Jordan algebra and $V_{1}=(V / \operatorname{Ker}(\alpha)) \oplus \operatorname{Ker}(\alpha)$ is the direct sum of ideals.

Now we show that $(V, \mu, \alpha) \cong\left(V_{1}, \mu_{1}, \alpha_{1}\right)$. According to the assumption, we have that $\operatorname{Im}(\alpha)$ is a Hom-ideal of $(V, \mu, \alpha)$. For any $x \in \operatorname{Ker}(\alpha) \cap \operatorname{Im}(\alpha)$, there exists $y \in V$ such that $x=\alpha(y)$. Then we have

$$
0=\alpha(x)=\alpha^{2}(y)=\alpha(y)=x,
$$

so $\operatorname{Ker}(\alpha) \cap \operatorname{Im}(\alpha)=\{0\}$. So for any $x \in V$, we have $x=x-\alpha(x)+\alpha(x)$, where $x-\alpha(x) \in \operatorname{Ker}(\alpha)$ and $\alpha(x) \in \operatorname{Im}(\alpha)$. Therefore, $V=\operatorname{Ker}(\alpha) \oplus \operatorname{Im}(\alpha)$.

Obviously, $\left(\operatorname{Im}(\alpha), \mu,\left.\alpha\right|_{\operatorname{Im}(\alpha)}\right)$ is a Hom-Jordan algebra.

Next we will show that $\left(\operatorname{Im}(\alpha), \mu,\left.\alpha\right|_{\operatorname{Im}(\alpha)} \cong(V / \operatorname{Ker}(\alpha), \bar{\mu}, \bar{\alpha})\right.$. Define the map $\varphi: V / \operatorname{Ker}(\alpha) \rightarrow \operatorname{Im}(\alpha)$ by $\varphi(\bar{x})=\alpha(x)$ for all $\bar{x} \in V / \operatorname{Ker}(\alpha)$. Obviously, $\varphi$ is bijective. For all $\bar{x}, \bar{y} \in V / \operatorname{Ker}(\alpha)$, we have

$$
\begin{gathered}
\varphi(\bar{\mu}(\bar{x}, \bar{y}))=\varphi(\overline{\mu(x, y)})=\alpha(\mu(x, y))=\mu(\alpha(x), \alpha(y))=\mu(\varphi(\bar{x}), \varphi(\bar{y})), \\
\varphi(\bar{\alpha}(\bar{x}))=\varphi(\overline{\alpha(x)})=\alpha^{2}(x)=\alpha(\varphi(\bar{x}))
\end{gathered}
$$

which implies that $\varphi \circ \bar{\alpha}=\alpha \circ \varphi$. So $\varphi$ is an isomorphism, i.e.,

$$
\left(\operatorname{Im}(\alpha), \mu,\left.\alpha\right|_{\operatorname{Im}(\alpha)}\right) \cong(V / \operatorname{Ker}(\alpha), \bar{\mu}, \bar{\alpha}) .
$$

Therefore, $V=\operatorname{Ker}(\alpha) \oplus \operatorname{Im}(\alpha) \cong(V / \operatorname{Ker}(\alpha)) \oplus \operatorname{Ker}(\alpha)$.

\section{Classification of Simple multiplicative Hom-Jordan algebras}

In this section we present a theorem about classification of simple multiplicative Hom-Jordan algebras. First, we give a construction of $n$-dimensional simple HomJordan algebras.

Theorem 4.1. There exist $n$-dimensional simple Hom-Jordan algebras for any $n \in \mathbb{Z}^{+}$.

Proof. When $n=1$, let $V=\mathbb{R}^{+}$over $\mathbb{R}$, i.e., $\mu: V \times V \rightarrow V, \mu(a, b)=\frac{1}{2}(a b+b a)$ for all $a, b \in \mathbb{R}$. It is obvious that $\operatorname{dim}(V)=1$. Take $\alpha=k \operatorname{id}_{\mathbb{R}}$ for $k \in \mathbb{R}$. Then $(V, \mu, \alpha)$ is a 1-dimensional Hom-Jordan algebra. Obviously, $(V, \mu, \alpha)$ is simple, since $(V, \mu, \alpha)$ has no nontrivial Hom-ideal and $\mu(V, V)=V$. 
When $n=2$, let $\left\{e_{0}, e_{1}\right\}$ be a basis of a 2 -dimensional vector space $V$ over $\mathbb{C}$. Define a bilinear symmetric binary operation $\mu: V \times V \rightarrow V$ as follows:

$$
\mu\left(e_{0}, e_{0}\right)=e_{0}, \quad \mu\left(e_{1}, e_{1}\right)=e_{1}, \quad \mu\left(e_{0}, e_{1}\right)=\mu\left(e_{1}, e_{0}\right)=e_{0}+e_{1} .
$$

Obviously, $\mu(V, V)=V$. Take $\alpha \in \operatorname{End}(V)$ such that

$$
\alpha\left(e_{0}\right)=p e_{0}, \quad \alpha\left(e_{1}\right)=q e_{1}, \quad p, q \in \mathbb{C} .
$$

One can verify that $(V, \mu, \alpha)$ is a 2-dimensional Hom-Jordan algebra. Next we will show that $(V, \mu, \alpha)$ is simple.

Suppose that $I$ is a nontrivial Hom-ideal of $(V, \mu, \alpha)$. Then there exists $0 \neq a=$ $t_{1} e_{0}+t_{2} e_{1} \in I$, where $t_{1}, t_{2} \in \mathbb{C}$. Then we have $\left(t_{1}+t_{2}\right) e_{0}+t_{2} e_{1}=\mu\left(a, e_{0}\right) \in I$, i.e., $\frac{t_{1}+t_{2}}{t_{1}}=\frac{t_{2}}{t_{2}} ; t_{1} e_{0}+\left(t_{1}+t_{2}\right) e_{1}=\mu\left(a, e_{1}\right) \in I$, i.e., $\frac{t_{2}}{t_{1}+t_{2}}=\frac{t_{1}}{t_{1}}$ since $\operatorname{dim}(I)=1$. So $t_{1}+t_{2}=t_{1}, t_{1}+t_{2}=t_{2}$, which imply that $t_{1}=0, t_{2}=0$. Hence, $I=0$, a contradiction. Therefore, $(V, \mu, \alpha)$ is a 2-dimensional simple Hom-Jordan algebra.

When $n \geq 3$, let $\left\{a_{\bar{i}} \mid i \in \mathbb{Z}_{n}\right\}$ be a basis of an $n$-dimensional vector space $V$ over $\mathbb{C}$. Define a bilinear symmetric binary operation $\mu: V \times V \rightarrow V$ as follows:

$$
\mu\left(a_{\bar{i}}, a_{\overline{i+1}}\right)=\mu\left(a_{\overline{i+1}}, a_{\bar{i}}\right)=a_{\overline{i+2}} ;
$$

all the others are zero. Then for any linear map $\alpha \in \operatorname{End}(V),(V, \mu, \alpha)$ is a HomJordan algebra.

Next, we prove that $(V, \mu, \alpha)$ is simple. Clearly, we have $\mu(V, V)=V$. Let $W$ be a nonzero Hom-ideal of $(V, \mu, \alpha)$; then there exists a nonzero element $x=$ $\sum_{i=0}^{n-1} x_{i} a_{\bar{i}} \in W$. Suppose that $x_{t} \neq 0$. Since $x_{t} a_{\overline{t+2}}=\mu\left(a_{\bar{t}}, \mu\left(a_{\overline{t-1}}, x\right)\right) \in W$, we have $a_{\overline{t+2}} \in W$, so $a_{\overline{n-2}}, a_{\overline{n-1}} \in W$. Then $a_{\overline{0}}=\mu\left(a_{\overline{n-2}}, a_{\overline{n-1}}\right) \in W, a_{\overline{1}}=$ $\mu\left(a_{\overline{n-1}}, a_{\overline{0}}\right) \in W$. Hence, we have all $a_{\bar{i}} \in W\left(i \in \mathbb{Z}_{n}\right)$. Therefore $W=V$ and $(V, \mu, \alpha)$ is simple.

According to Theorem 3.5 (1) and Corollary 2.13, the dimension of a simple multiplicative Hom-Jordan algebra can only be an integer multiple of dimensions of simple Jordan algebras.

Also by Theorem 3.5 (1) and Corollary 2.13 in order to classify simple multiplicative Hom-Jordan algebras, we just classify automorphisms on their induced Jordan algebras; in particular, automorphisms on semi-simple Jordan algebras which are direct sum of finite isomorphic simple ideals.

Theorem 4.2. Let $J$ be a semi-simple Jordan algebra such that its $n$ simple ideals are mutually isomorphic; moreover, $J$ can be generated by its automorphism $\alpha$ (or $\beta$ ) and any simple ideal, and $\alpha_{n}\left(\beta_{n}\right)$ leaves each simple ideal of $J$ invariant, where $\alpha_{n}=\alpha^{n}$ and $\beta_{n}=\beta^{n}$. Then there exists an automorphism $\varphi$ on $J$ satisfying $\varphi \circ \alpha=\beta \circ \varphi$ if and only if there exists an automorphism $\psi$ on the simple ideal of $J$ satisfying $\psi \circ \alpha^{n}=\beta^{n} \circ \psi$.

Proof. Let $J_{1}$ be a simple ideal of $J$. Since $\alpha_{n}$ (or $\beta_{n}$ ) leaves each simple ideal of $J$ invariant, we have $\alpha^{n}\left(J_{1}\right)=J_{1}$ (or $\left.\beta^{n}\left(J_{1}\right)=J_{1}\right)$ and we have

$$
\begin{aligned}
J & =J_{1} \oplus \alpha\left(J_{1}\right) \oplus \cdots \oplus \alpha^{n-1}\left(J_{1}\right) \\
\text { or } \quad J & =J_{1} \oplus \beta\left(J_{1}\right) \oplus \cdots \oplus \beta^{n-1}\left(J_{1}\right),
\end{aligned}
$$


since $J$ can be generated by its automorphism $\alpha$ (or $\beta$ ) and any simple ideal.

Choose a basis $x=\left(x_{1}, x_{2}, \ldots, x_{m}\right)$ of $J_{1}$; then

$$
x^{\prime}=\left(x, \alpha(x), \alpha^{2}(x), \ldots, \alpha^{n-1}(x)\right) \quad \text { and } \quad x^{\prime \prime}=\left(x, \beta(x), \beta^{2}(x), \ldots, \beta^{n-1}(x)\right)
$$

are both bases of $J$. Let $\alpha\left(x^{\prime}\right)=x^{\prime} A, \beta\left(x^{\prime \prime}\right)=x^{\prime \prime} B$; then

$$
A=\left(\begin{array}{ccccc}
0 & & & & A_{1} \\
I & 0 & & & \\
& I & 0 & & \\
& & \ddots & \ddots & \\
& & & I & 0
\end{array}\right), \quad B=\left(\begin{array}{ccccc}
0 & & & & B_{1} \\
I & 0 & & & \\
& I & 0 & & \\
& & \ddots & \ddots & \\
& & & I & 0
\end{array}\right) \text {, }
$$

where $\alpha_{n}(x)=x A_{1}, \beta_{n}(x)=x B_{1}$.

If there exists an automorphism $\psi$ on $J_{1}$ such that $\psi \circ \alpha_{n}=\beta_{n} \circ \psi$, then $M A_{1}=$ $B_{1} M$, where $M$ is defined by $\psi(x)=x M$. Define $\varphi\left(x^{\prime}\right)=x^{\prime \prime} \operatorname{diag}(M, \ldots, M)$. Then we have $\varphi\left(x^{\prime}\right)=\left(\psi(x), \beta(\psi(x)), \ldots, \beta^{n-1}(\psi(x))\right)$. It is easy to verify that $\varphi$ is an automorphism, since $\psi$ is an automorphism. Moreover,

$$
\begin{aligned}
& \varphi \circ \alpha\left(x^{\prime}\right)=x^{\prime \prime}\left(\begin{array}{ccccc}
M & & & & \\
& M & & & \\
& & \ddots & & \\
& & & \ddots & \\
& & & & M
\end{array}\right)\left(\begin{array}{ccccc}
0 & & & & A_{1} \\
I & 0 & & & \\
& I & 0 & & \\
& & \ddots & \ddots & \\
& & & I & 0
\end{array}\right) \\
& =x^{\prime \prime}\left(\begin{array}{ccccc}
0 & \cdots & \cdots & 0 & M A_{1} \\
M & 0 & & & \\
& M & 0 & & \\
& & & \ddots & \\
& & & M & 0
\end{array}\right) \text {, } \\
& \beta \circ \varphi\left(x^{\prime}\right)=x^{\prime \prime}\left(\begin{array}{ccccc}
0 & & & & B_{1} \\
I & 0 & & & \\
& I & 0 & & \\
& & \ddots & \ddots & \\
& & & I & 0
\end{array}\right)\left(\begin{array}{lllll}
M & & & & \\
& M & & & \\
& & \ddots & & \\
& & & \ddots & \\
& & & & M
\end{array}\right) \\
& =x^{\prime \prime}\left(\begin{array}{ccccc}
0 & \cdots & \cdots & 0 & B_{1} M \\
M & 0 & & & \\
& M & 0 & & \\
& & & \ddots & \\
& & & M & 0
\end{array}\right) \text {. }
\end{aligned}
$$

Note that $M A_{1}=B_{1} M$. We have $\varphi \circ \alpha=\beta \circ \varphi$.

Now suppose that there exists an automorphism $\varphi$ on $J$ satisfying $\varphi \circ \alpha=\beta \circ \varphi$. According to the proof of Lemma 3.4 , there exists $0 \leq i \leq n-1$ such that $\varphi\left(J_{1}\right)=$ 
$\beta^{i}\left(J_{1}\right)$. Then $\varphi \circ \alpha^{j}\left(J_{1}\right)=\beta^{i+j}\left(J_{1}\right)(0 \leq i, j \leq n-1)$. Let $\varphi(x)=\beta^{i}(x) M_{1}$; then $\varphi\left(x^{\prime}\right)=x^{\prime \prime} M$, where $M$ is

$$
\left(\begin{array}{lllll}
M_{1} & & & & \\
& M_{1} & & & \\
& & \ddots & & \\
& & & \ddots & \\
& & & & M_{1}
\end{array}\right) \quad(i=0)
$$

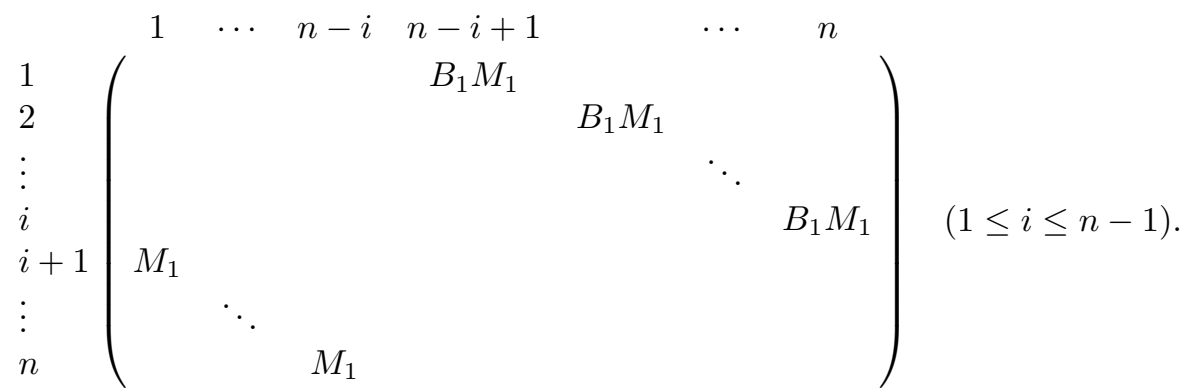

Defining $\psi(x)=x M_{1}$, we have

$\varphi\left(x^{\prime}\right)= \begin{cases}\left(\psi(x), \beta(\psi(x)), \ldots, \beta^{n-1}(\psi(x))\right), & (i=0) ; \\ \left(\beta^{i}(\psi(x)), \ldots, \beta^{n-1}(\psi(x)), \beta^{n}(\psi(x)), \ldots, \beta^{n+i-1}(\psi(x))\right), & (1 \leq i \leq n-1) .\end{cases}$

Therefore, $\psi$ is an automorphism on $J_{1}$, since $\varphi$ is an automorphism on $J$. Moreover, we have $\psi \circ \alpha^{n}=\beta^{n} \circ \psi$, since $\varphi \circ \alpha=\beta \circ \varphi$.

By Theorem 4.2, it is obvious that two simple multiplicative Hom-Jordan algebras $\left(V_{1}, \mu_{1}, \alpha\right)$ and $\left(V_{2}, \mu_{2}, \beta\right)$ are isomorphic if and only if the automorphisms $\alpha^{n}$ and $\beta^{n}$ on two simple ideals (as simple Jordan algebras) of the corresponding induced Jordan algebras are conjugate.

Combining Corollary 2.13 Theorem 3.5 (1) and Theorem 4.2 we get the following theorem.

Theorem 4.3. All finite-dimensional simple multiplicative Hom-Jordan algebras can be denoted as $\left(X, n, \Gamma_{\alpha}\right)$, where $X$ represents the type of the simple ideal (as the simple Jordan algebra) of the corresponding induced Jordan algebras, $n$ represents numbers of simple ideals, and $\Gamma_{\alpha}$ represents the set of conjugate classes of the automorphism $\alpha^{n}$ on the simple Jordan algebra $X$, i.e., $\Gamma_{\alpha}=\left\{\phi \circ \alpha^{n} \circ \phi^{-1} \mid \phi \in\right.$ $\operatorname{Aut}(X)\}$.

Example 4.4. Suppose that $V$ is a 2-dimensional vector space with basis $\left\{e_{1}, e_{2}\right\}$. Define a bilinear map $\mu: V \times V \rightarrow V$ by

$$
\left\{\begin{array}{l}
\mu\left(e_{1}, e_{1}\right)=e_{2}, \\
\mu\left(e_{2}, e_{2}\right)=e_{1}, \\
\mu\left(e_{1}, e_{2}\right)=\mu\left(e_{2}, e_{1}\right)=0,
\end{array}\right.
$$


and a linear map $\alpha: V \rightarrow V$ by

$$
\left\{\begin{array}{l}
\alpha\left(e_{1}\right)=e_{2}, \\
\alpha\left(e_{2}\right)=e_{1} .
\end{array}\right.
$$

Then $(V, \mu, \alpha)$ is a simple multiplicative Hom-Jordan algebra. Moreover, its induced Jordan algebra is $\left(V, \mu^{\prime}\right)$, where $\mu^{\prime}: V \times V \rightarrow V$ satisfies

$$
\left\{\begin{array}{l}
\mu^{\prime}\left(e_{1}, e_{1}\right)=e_{1}, \\
\mu^{\prime}\left(e_{2}, e_{2}\right)=e_{2}, \\
\mu^{\prime}\left(e_{1}, e_{2}\right)=\mu\left(e_{2}, e_{1}\right)=0 .
\end{array}\right.
$$

$\left(V, \mu^{\prime}\right)$ is semi-simple and has the decomposition into simple ideals $V=V_{1} \oplus V_{2}$, where $V_{1}$ and $V_{2}$ are simple ideals generated by $e_{1}$ and $e_{2}$, respectively. Moreover, we get that $V_{1}$ is isomorphic to $V_{2}$.

According to Theorem $4.3(V, \mu, \alpha)$ can be denoted as $\left(V_{1}, 2, \alpha^{2}\right)$ or $\left(V_{2}, 2, \alpha^{2}\right)$.

\section{Bimodules of Simple multiplicative Hom-Jordan Algebras}

In this section we mainly study bimodules of simple multiplicative Hom-Jordan algebras. We give a theorem on relationships between bimodules of Jordan-type Hom-Jordan algebras and modules of their induced Jordan algebras. Moreover, some propositions about bimoudles of simple multiplicative Hom-Jordan algebras are also obtained.

Definition $5.1([3])$. Let $(V, \mu, \alpha)$ be a Hom-Jordan algebra. A $V$-bimodule is a Hom-module $\left(W, \alpha_{W}\right)$ that comes equipped with a left structure map $\rho_{l}: V \otimes W \rightarrow$ $W\left(\rho_{l}(a \otimes w)=a \cdot w\right)$ and a right structure map $\rho_{r}: W \otimes V \rightarrow W\left(\rho_{l}(w \otimes a)=w \cdot a\right)$ such that the following conditions hold for all $a, b, c \in V, w \in W$ :

(1) $\rho_{r} \circ \tau_{1}=\rho_{l}$, where $\tau_{1}: V \otimes W \rightarrow W \otimes V, a \otimes w \mapsto w \otimes a$;

(2) $\alpha_{W}(w \cdot a) \cdot \alpha(\mu(b, c))+\alpha_{W}(w \cdot b) \cdot \alpha(\mu(c, a))+\alpha_{W}(w \cdot c) \cdot \alpha(\mu(a, b))=$ $\left(\alpha_{W}(w) \cdot \mu(b, c)\right) \cdot \alpha^{2}(a)+\left(\alpha_{W}(w) \cdot \mu(c, a)\right) \cdot \alpha^{2}(b)+\left(\alpha_{W}(w) \cdot \mu(a, b)\right) \cdot \alpha^{2}(c) ;$

(3) $\alpha_{W}(w \cdot a) \cdot \alpha(\mu(b, c))+\alpha_{W}(w \cdot b) \cdot \alpha(\mu(c, a))+\alpha_{W}(w \cdot c) \cdot \alpha(\mu(a, b))=$ $((w \cdot a) \cdot \alpha(b)) \cdot \alpha^{2}(c)+((w \cdot c) \cdot \alpha(b)) \cdot \alpha^{2}(a)+\mu(\mu(a, c), \alpha(b)) \cdot \alpha_{W}^{2}(w)$.

Example 5.2. In [3], suppose that $(V, \mu, \alpha)$ is a Hom-Jordan algebra; then $(V, \alpha)$ is a $V$-bimodule where the structure maps are $\rho_{l}=\rho_{r}=\mu$.

Next, we construct an example over a field of prime characteristic.

Example 5.3. Let $(V, \mu, \alpha)$ be the Hom-Jordan algebra in Example 4.4 and $W$ a 1-dimensional vector space with basis $\left\{w_{1}\right\}$. Define $\alpha_{W}: W \rightarrow W$ to be a linear map by $\alpha_{W}\left(w_{1}\right)=w_{1}$. Define two linear maps: $\rho_{l}: V \otimes W \rightarrow W$ by

and $\rho_{r}: W \otimes V \rightarrow W$ by

$$
\left\{\begin{array}{l}
\rho_{l}\left(e_{1}, w_{1}\right)=w_{1}, \\
\rho_{l}\left(e_{2}, w_{1}\right)=w_{1}
\end{array}\right.
$$

$$
\left\{\begin{array}{l}
\rho_{l}\left(w_{1}, e_{1}\right)=w_{1}, \\
\rho_{l}\left(w_{1}, e_{2}\right)=w_{1} .
\end{array}\right.
$$


Then $\left(W, \alpha_{W}\right)$ is a $V$-bimodule with the structure maps defined as above when the characteristic of the ground field is two.

Definition $5.4([8])$. A Jordan module is a system consisting of a vector space $V$, a Jordan algebra $J$, and two compositions $x \cdot a, a \cdot x$ for $x$ in $V, a$ in $J$ which are bilinear and satisfy

(i) $a \cdot x=x \cdot a$;

(ii) $(x \cdot a) \cdot(b \circ c)+(x \cdot b) \cdot(c \circ a)+(x \cdot c) \cdot(a \circ b)=(x \cdot(b \circ c)) \cdot a+(x \cdot(c \circ a)) \cdot b+(x \cdot(a \circ b)) \cdot c$;

(iii) $(((x \cdot a) \cdot b) \cdot c)+(((x \cdot c) \cdot b) \cdot a)+x \cdot(a \circ c \circ b)=(x \cdot a) \cdot(b \circ c)+(x \cdot b) \cdot(c \circ a)+(x \cdot c) \cdot(a \circ b)$, where $x \in V, a, b, c \in J$, and $a_{1} \circ a_{2} \circ a_{3}$ stands for $\left(\left(a_{1} \circ a_{2}\right) \circ a_{3}\right)$.

Theorem 5.5. Let $(V, \mu, \alpha)$ be a Jordan-type Hom-Jordan algebra with $\left(V, \mu^{\prime}\right)$ the induced Jordan algebra.

(1) Let $\left(W, \alpha_{W}\right)$ be a $V$-bimodule of $(V, \mu, \alpha)$ with $\rho_{l}\left(\rho_{r}\right)$ the left structure map (respectively, the right structure map). Suppose that $\alpha_{W}$ is invertible and satisfies $\alpha_{W}(w \cdot a)=\alpha_{W}(w) \cdot \alpha(a)$ for all $a \in V, w \in W$. Then $W$ is a module of the induced Jordan algebra $\left(V, \mu^{\prime}\right)$ with two compositions $w \cdot^{\prime} a=\alpha_{W}^{-1}(w \cdot a)$ and $a \cdot^{\prime} w=\alpha_{W}^{-1}(a \cdot w)$ for all $a \in V, w \in W$.

(2) Let $W$ be a module of the induced Jordan algebra $\left(V, \mu^{\prime}\right)$ with two compositions $w \cdot^{\prime} a$ and $a \cdot^{\prime} w$ for all $a \in V, w \in W$. If there exists $\alpha_{W} \in \operatorname{End}(W)$ such that $\alpha_{W}\left(w \cdot^{\prime} a\right)=\alpha_{W}(w) \cdot^{\prime} \alpha(a)$ for all $a \in V, w \in W$, then $\left(W, \alpha_{W}\right)$ is a V-bimodule of $(V, \mu, \alpha)$ with the left structure map $\rho_{l}: V \otimes W \rightarrow W\left(\rho_{l}(a \otimes w)=\alpha_{W}\left(a \cdot^{\prime} w\right)\right)$ and the right structure map $\rho_{r}: W \otimes V \rightarrow W\left(\rho_{r}(w \otimes a)=\alpha_{W}\left(w \cdot{ }^{\prime} a\right)\right)$.

Proof. (1) For any $x \in W, a, b, c \in V$, we have

$$
\begin{aligned}
& a \cdot^{\prime} x= \alpha_{W}^{-1}(a \cdot x)=\alpha_{W}^{-1}\left(\rho_{l}(a \otimes x)\right)=\alpha_{W}^{-1}\left(\rho_{r} \circ \tau_{1}(a \otimes x)\right)=\alpha_{W}^{-1}(x \cdot a)=x \cdot^{\prime} a ; \\
&\left(x \cdot^{\prime} a\right) \cdot^{\prime} \mu^{\prime}(b, c)+\left(x \cdot^{\prime} b\right) \cdot^{\prime} \mu^{\prime}(c, a)+\left(x \cdot^{\prime} c\right) \cdot^{\prime} \mu^{\prime}(a, b) \\
&=\alpha_{W}^{-1}\left(\alpha_{W}^{-1}(x \cdot a) \cdot \alpha^{-1}(\mu(b, c))\right)+\alpha_{W}^{-1}\left(\alpha_{W}^{-1}(x \cdot b) \cdot \alpha^{-1}(\mu(c, a))\right) \\
&+\alpha_{W}^{-1}\left(\alpha_{W}^{-1}(x \cdot c) \cdot \alpha^{-1}(\mu(a, b))\right) \\
&= \alpha_{W}^{-3}\left(\alpha_{W}^{2}\left(\alpha_{W}^{-1}(x \cdot a) \cdot \alpha^{-1}(\mu(b, c))\right)+\alpha_{W}^{2}\left(\alpha_{W}^{-1}(x \cdot b) \cdot \alpha^{-1}(\mu(c, a))\right)\right. \\
&\left.+\alpha_{W}^{2}\left(\alpha_{W}^{-1}(x \cdot c) \cdot \alpha^{-1}(\mu(a, b))\right)\right) \\
&= \alpha_{W}^{-3}\left(\alpha_{W}((x \cdot a) \cdot \mu(b, c))+\alpha_{W}((x \cdot b) \cdot \mu(c, a))+\alpha_{W}((x \cdot c) \cdot \mu(a, b))\right) \\
&= \alpha_{W}^{-3}\left(\alpha_{W}(x \cdot a) \cdot \alpha(\mu(b, c))+\alpha_{W}(x \cdot b) \cdot \alpha(\mu(c, a))+\alpha_{W}(x \cdot c) \cdot \alpha(\mu(a, b))\right) \\
&= \alpha_{W}^{-3}\left(\left(\alpha_{W}(x) \cdot \mu(b, c)\right) \cdot \alpha^{2}(a)+\left(\alpha_{W}(x) \cdot \mu(c, a)\right) \cdot \alpha^{2}(b)\right. \\
&\left.+\left(\alpha_{W}(x) \cdot \mu(a, b)\right) \cdot \alpha^{2}(c)\right) \\
&= \alpha_{W}^{-3}\left(\alpha_{W}\left(x \cdot \alpha^{-1}(\mu(b, c))\right) \cdot \alpha^{2}(a)+\alpha_{W}\left(x \cdot \alpha^{-1}(\mu(c, a))\right) \cdot \alpha^{2}(b)\right. \\
&\left.+\alpha_{W}\left(x \cdot \alpha^{-1}(\mu(a, b))\right) \cdot \alpha^{2}(c)\right) \\
&= \alpha_{W}^{-3}\left(\alpha_{W}^{2}\left(x \cdot{ }^{\prime} \mu^{\prime}(b, c)\right) \cdot \alpha^{2}(a)+\alpha_{W}^{2}\left(x \cdot{ }^{\prime} \mu^{\prime}(c, a)\right) \cdot \alpha^{2}(b)\right. \\
&\left.+\alpha_{W}^{2}\left(x \cdot^{\prime} \mu^{\prime}(a, b)\right) \cdot \alpha^{2}(c)\right)
\end{aligned}
$$




$$
\begin{aligned}
& =\alpha_{W}^{-3}\left(\alpha_{W}^{2}\left(\left(x \cdot^{\prime} \mu^{\prime}(b, c)\right) \cdot a\right)+\alpha_{W}^{2}\left(\left(x \cdot^{\prime} \mu^{\prime}(c, a)\right) \cdot b\right)+\alpha_{W}^{2}\left(\left(x \cdot^{\prime} \mu^{\prime}(a, b)\right) \cdot c\right)\right) \\
& =\alpha_{W}^{-1}\left(\left(x \cdot^{\prime} \mu^{\prime}(b, c)\right) \cdot a\right)+\alpha_{W}^{-1}\left(\left(x \cdot^{\prime} \mu^{\prime}(c, a)\right) \cdot b\right)+\alpha_{W}^{-1}\left(\left(x \cdot^{\prime} \mu^{\prime}(a, b)\right) \cdot c\right) \\
& =\left(x \cdot{ }^{\prime} \mu^{\prime}(b, c)\right) \cdot^{\prime} a+\left(x \cdot{ }^{\prime} \mu^{\prime}(c, a)\right) \cdot{ }^{\prime} b+\left(x \cdot{ }^{\prime} \mu^{\prime}(a, b)\right) \cdot{ }^{\prime} c \text {; } \\
& \left(x \cdot^{\prime} a\right) \cdot^{\prime} \mu^{\prime}(b, c)+\left(x \cdot^{\prime} b\right) \cdot^{\prime} \mu^{\prime}(c, a)+\left(x \cdot^{\prime} c\right) \cdot^{\prime} \mu^{\prime}(a, b) \\
& =\alpha_{W}^{-3}\left(\alpha_{W}(x \cdot a) \cdot \alpha(\mu(b, c))+\alpha_{W}(x \cdot b) \cdot \alpha(\mu(c, a))+\alpha_{W}(x \cdot c) \cdot \alpha(\mu(a, b))\right) \\
& =\alpha_{W}^{-3}\left(((x \cdot a) \cdot \alpha(b)) \cdot \alpha^{2}(c)+((x \cdot c) \cdot \alpha(b)) \cdot \alpha^{2}(a)+\mu(\mu(a, c), \alpha(b)) \cdot \alpha_{W}^{2}(x)\right) \\
& =\alpha_{W}^{-3}\left(\left(\alpha_{W}\left(x \cdot^{\prime} a\right) \cdot \alpha(b)\right) \cdot \alpha^{2}(c)+\left(\alpha_{W}\left(x \cdot^{\prime} c\right) \cdot \alpha(b)\right) \cdot \alpha^{2}(a)\right. \\
& \left.+\alpha\left(\mu^{\prime}\left(\alpha\left(\mu^{\prime}(a, c)\right), \alpha(b)\right)\right) \cdot \alpha_{W}^{2}(x)\right) \\
& =\alpha_{W}^{-3}\left(\alpha_{W}\left(\left(x \cdot^{\prime} a\right) \cdot b\right) \cdot \alpha^{2}(c)+\alpha_{W}\left(\left(x \cdot^{\prime} c\right) \cdot b\right) \cdot \alpha^{2}(a)\right. \\
& \left.+\alpha^{2}\left(\mu^{\prime}\left(\mu^{\prime}(a, c), b\right)\right) \cdot \alpha_{W}^{2}(x)\right) \\
& =\alpha_{W}^{-3}\left(\alpha_{W}^{2}\left(\left(x \cdot^{\prime} a\right) \cdot^{\prime} b\right) \cdot \alpha^{2}(c)+\alpha_{W}^{2}\left(\left(x \cdot^{\prime} c\right) \cdot^{\prime} b\right) \cdot \alpha^{2}(a)\right. \\
& \left.+\alpha^{2}\left(\mu^{\prime}\left(\mu^{\prime}(a, c), b\right)\right) \cdot \alpha_{W}^{2}(x)\right) \\
& =\alpha_{W}^{-3}\left(\alpha_{W}^{2}\left(\left(\left(x \cdot^{\prime} a\right) \cdot^{\prime} b\right) \cdot c\right)+\alpha_{W}^{2}\left(\left(\left(x \cdot^{\prime} c\right) \cdot^{\prime} b\right) \cdot a\right)+\alpha_{W}^{2}\left(\mu^{\prime}\left(\mu^{\prime}(a, c), b\right) \cdot x\right)\right) \\
& =\alpha_{W}^{-1}\left(\left(\left(x \cdot^{\prime} a\right) \cdot^{\prime} b\right) \cdot c\right)+\alpha_{W}^{-1}\left(\left(\left(x \cdot^{\prime} c\right) \cdot^{\prime} b\right) \cdot a\right)+\alpha_{W}^{-1}\left(\mu^{\prime}\left(\mu^{\prime}(a, c), b\right) \cdot x\right) \\
& =\left(\left(x \cdot^{\prime} a\right) \cdot^{\prime} b\right) \cdot^{\prime} c+\left(\left(x \cdot^{\prime} c\right) \cdot^{\prime} b\right) \cdot^{\prime} a+\mu^{\prime}\left(\mu^{\prime}(a, c), b\right) \cdot^{\prime} x .
\end{aligned}
$$

Therefore, $W$ is a module of the induced Jordan algebra $\left(V, \mu^{\prime}\right)$.

(2) For any $w \in W, a, b, c \in V$, we have

$$
\rho_{r} \circ \tau_{1}(a \otimes w)=\alpha_{W}\left(w \cdot^{\prime} a\right)=\alpha_{W}\left(a \cdot^{\prime} w\right)=\rho_{l}(a \otimes w),
$$

which implies that $\rho_{r} \circ \tau_{1}=\rho_{l}$. Then we see that

$$
\begin{aligned}
& \alpha_{W}(w \cdot a) \cdot \alpha(\mu(b, c))+\alpha_{W}(w \cdot b) \cdot \alpha(\mu(c, a))+\alpha_{W}(w \cdot c) \cdot \alpha(\mu(a, b)) \\
&= \alpha_{W}\left(\alpha_{W}^{2}\left(w \cdot^{\prime} a\right) \cdot^{\prime} \alpha^{2}\left(\mu^{\prime}(b, c)\right)\right)+\alpha_{W}\left(\alpha_{W}^{2}\left(w \cdot^{\prime} b\right) \cdot^{\prime} \alpha^{2}\left(\mu^{\prime}(c, a)\right)\right) \\
&+\alpha_{W}\left(\alpha_{W}^{2}\left(w \cdot^{\prime} c\right) \cdot^{\prime} \alpha^{2}\left(\mu^{\prime}(a, b)\right)\right) \\
&= \alpha_{W}\left(\alpha_{W}^{2}\left(\left(w \cdot^{\prime} a\right) \cdot^{\prime} \mu^{\prime}(b, c)\right)+\alpha_{W}^{2}\left(\left(w \cdot^{\prime} b\right) \cdot^{\prime} \mu^{\prime}(c, a)\right)+\alpha_{W}^{2}\left(\left(w \cdot^{\prime} c\right) \cdot^{\prime} \mu^{\prime}(a, b)\right)\right) \\
&= \alpha_{W}^{3}\left(\left(w \cdot^{\prime} a\right) \cdot^{\prime} \mu^{\prime}(b, c)+\left(w \cdot^{\prime} b\right) \cdot^{\prime} \mu^{\prime}(c, a)+\left(w \cdot^{\prime} c\right) \cdot^{\prime} \mu^{\prime}(a, b)\right) \\
&= \alpha_{W}^{3}\left(\left(w \cdot^{\prime} \mu^{\prime}(b, c)\right) \cdot^{\prime} a+\left(w \cdot^{\prime} \mu^{\prime}(c, a)\right) \cdot^{\prime} b+\left(w \cdot^{\prime} \mu^{\prime}(a, b)\right) \cdot^{\prime} c\right) \\
&= \alpha_{W}^{2}\left(\alpha_{W}\left(w \cdot^{\prime} \mu^{\prime}(b, c)\right) \cdot^{\prime} \alpha(a)\right)+\alpha_{W}^{2}\left(\alpha_{W}\left(w \cdot^{\prime} \mu^{\prime}(c, a)\right) \cdot^{\prime} \alpha(b)\right) \\
&+\alpha_{W}^{2}\left(\alpha_{W}\left(w \cdot^{\prime} \mu^{\prime}(a, b)\right) \cdot^{\prime} \alpha(c)\right) \\
&= \alpha_{W}\left(\alpha_{W}^{2}\left(w \cdot^{\prime} \mu^{\prime}(b, c)\right) \cdot^{\prime} \alpha^{2}(a)\right)+\alpha_{W}\left(\alpha_{W}^{2}\left(w \cdot^{\prime} \mu^{\prime}(c, a)\right) \cdot^{\prime} \alpha^{2}(b)\right) \\
&+\alpha_{W}\left(\alpha_{W}^{2}\left(w \cdot^{\prime} \mu^{\prime}(a, b)\right) \cdot^{\prime} \alpha^{2}(c)\right) \\
&= \alpha_{W}^{2}\left(w \cdot^{\prime} \mu^{\prime}(b, c)\right) \cdot \alpha^{2}(a)+\alpha_{W}^{2}\left(w \cdot^{\prime} \mu^{\prime}(c, a)\right) \cdot \alpha^{2}(b)+\alpha_{W}^{2}\left(w \cdot^{\prime} \mu^{\prime}(a, b)\right) \cdot \alpha^{2}(c) \\
&= \alpha_{W}\left(\alpha_{W}(w) \cdot^{\prime} \alpha\left(\mu^{\prime}(b, c)\right)\right) \cdot \alpha^{2}(a)+\alpha_{W}\left(\alpha_{W}(w) \cdot^{\prime} \alpha\left(\mu^{\prime}(c, a)\right)\right) \cdot \alpha^{2}(b) \\
&+\alpha_{W}\left(\alpha_{W}(w) \cdot^{\prime} \alpha\left(\mu^{\prime}(a, b)\right)\right) \cdot \alpha^{2}(c)
\end{aligned}
$$




$$
\begin{aligned}
&=\left(\alpha_{W}(w) \cdot \mu(b, c)\right) \cdot \alpha^{2}(a)+\left(\alpha_{W}(w) \cdot \mu(c, a)\right) \cdot \alpha^{2}(b)+\left(\alpha_{W}(w) \cdot \mu(a, b)\right) \cdot \alpha^{2}(c) ; \\
& \alpha_{W}(w \cdot a) \cdot \alpha(\mu(b, c))+\alpha_{W}(w \cdot b) \cdot \alpha(\mu(c, a))+\alpha_{W}(w \cdot c) \cdot \alpha(\mu(a, b)) \\
&=\alpha_{W}^{3}\left(\left(w \cdot^{\prime} a\right) \cdot^{\prime} \mu^{\prime}(b, c)+\left(w \cdot^{\prime} b\right) \cdot^{\prime} \mu^{\prime}(c, a)+\left(w \cdot^{\prime} c\right) \cdot^{\prime} \mu^{\prime}(a, b)\right) \\
&=\alpha_{W}^{3}\left(\left(\left(w \cdot^{\prime} a\right) \cdot^{\prime} b\right) \cdot^{\prime} c+\left(\left(w \cdot^{\prime} c\right) \cdot^{\prime} b\right) \cdot^{\prime} a+\mu^{\prime}\left(\mu^{\prime}(a, c), b\right) \cdot^{\prime} w\right) \\
&=\alpha_{W}^{2}\left(\alpha_{W}\left(\left(w \cdot^{\prime} a\right) \cdot^{\prime} b\right) \cdot^{\prime} \alpha(c)\right)+\alpha_{W}^{2}\left(\alpha_{W}\left(\left(w \cdot^{\prime} c\right) \cdot^{\prime} b\right) \cdot^{\prime} \alpha(a)\right) \\
&+\alpha_{W}^{2}\left(\alpha\left(\mu^{\prime}\left(\mu^{\prime}(a, c), b\right)\right) \cdot^{\prime} \alpha_{W}(w)\right) \\
&= \alpha_{W}\left(\alpha_{W}^{2}\left(\left(w \cdot^{\prime} a\right) \cdot^{\prime} b\right) \cdot^{\prime} \alpha^{2}(c)\right)+\alpha_{W}\left(\alpha_{W}^{2}\left(\left(w \cdot^{\prime} c\right) \cdot^{\prime} b\right) \cdot^{\prime} \alpha^{2}(a)\right) \\
&+\alpha_{W}\left(\alpha^{2}\left(\mu^{\prime}\left(\mu^{\prime}(a, c), b\right)\right) \cdot^{\prime} \alpha_{W}^{2}(w)\right) \\
&= \alpha_{W}^{2}\left(\left(w \cdot^{\prime} a\right) \cdot^{\prime} b\right) \cdot \alpha^{2}(c)+\alpha_{W}^{2}\left(\left(w \cdot^{\prime} c\right) \cdot^{\prime} b\right) \cdot \alpha^{2}(a)+\alpha^{2}\left(\mu^{\prime}\left(\mu^{\prime}(a, c), b\right)\right) \cdot \alpha_{W}^{2}(w) \\
&= \alpha_{W}\left(\alpha_{W}\left(w \cdot^{\prime} a\right) \cdot^{\prime} \alpha(b)\right) \cdot \alpha^{2}(c)+\alpha_{W}\left(\alpha_{W}\left(w \cdot^{\prime} c\right) \cdot^{\prime} \alpha(b)\right) \cdot \alpha^{2}(a) \\
&+\alpha\left(\mu^{\prime}\left(\alpha\left(\mu^{\prime}(a, c)\right), \alpha(b)\right)\right) \cdot \alpha_{W}^{2}(w) \\
&=((w \cdot a) \cdot \alpha(b)) \cdot \alpha^{2}(c)+((w \cdot c) \cdot \alpha(b)) \cdot \alpha^{2}(a)+\mu(\mu(a, c), \alpha(b)) \cdot \alpha_{W}^{2}(w) .
\end{aligned}
$$

Therefore, $\left(W, \alpha_{W}\right)$ is a $V$-bimodule of $(V, \mu, \alpha)$.

Definition 5.6. For a bimodule $\left(W, \alpha_{W}\right)$ of a Hom-Jordan algebra $(V, \mu, \alpha)$, if a subspace $W_{0} \subseteq W$ satisfies the conditions $\rho_{l}(a \otimes w) \in W_{0}$ for any $a \in V$, $w \in W_{0}$ and $\alpha_{W}\left(W_{0}\right) \subseteq W_{0}$, then $\left(W_{0},\left.\alpha_{W}\right|_{W_{0}}\right)$ is called a $V$-submodule of $\left(W, \alpha_{W}\right)$. A bimodule $\left(W, \alpha_{W}\right)$ of a Hom-Jordan algebra $(V, \mu, \alpha)$ is called irreducible if it has precisely two $V$-submodules (itself and 0 ) and is called completely reducible if $W=W_{1} \oplus W_{2} \oplus \cdots \oplus W_{s}$, where $\left(W_{i},\left.\alpha_{W}\right|_{W_{i}}\right)$ are irreducible $V$-submodules.

Proposition 5.7. Suppose that $\left(W, \alpha_{W}\right)$ is a bimodule of the simple multiplicative Hom-Jordan algebra $(V, \mu, \alpha)$ with $\alpha_{W}(a \cdot w)=\alpha(a) \cdot \alpha_{W}(w)$ for all $a \in V, w \in W$. Then, $\operatorname{Ker}\left(\alpha_{W}\right)$ and $\operatorname{Im}\left(\alpha_{W}\right)$ are submodules of $W$ for $(V, \mu, \alpha)$. Moreover, we have an isomorphism of $(V, \mu, \alpha)$-modules $\overline{\alpha_{W}}: W / \operatorname{Ker}\left(\alpha_{W}\right) \rightarrow \operatorname{Im}\left(\alpha_{W}\right)$.

Proof. For any $w \in \operatorname{Ker}\left(\alpha_{W}\right)$, we have

$$
\alpha_{W}(a \cdot w)=\alpha(a) \cdot \alpha_{W}(w)=0, \quad \forall a \in V,
$$

which implies that $a \cdot w \in \operatorname{Ker}\left(\alpha_{W}\right)$. Obviously, $\alpha_{W}\left(\operatorname{Ker}\left(\alpha_{W}\right)\right) \subseteq \operatorname{Ker}\left(\alpha_{W}\right)$. Therefore, $\operatorname{Ker}\left(\alpha_{W}\right)$ is a submodule of $W$ for $(V, \mu, \alpha)$.

For any $w \in \operatorname{Im}\left(\alpha_{W}\right), a \in V$, there exist $u \in W$ and $\tilde{a} \in V$ such that $w=\alpha_{W}(u)$ and $a=\alpha(\tilde{a})$. Then

$$
a \cdot w=\alpha(\tilde{a}) \cdot \alpha_{W}(u)=\alpha_{W}(\tilde{a} \cdot u) \in \operatorname{Im}\left(\alpha_{W}\right) .
$$

Note that $\alpha_{W}\left(\operatorname{Im}\left(\alpha_{W}\right)\right) \subseteq \operatorname{Im}\left(\alpha_{W}\right)$. We obtain that $\operatorname{Im}\left(\alpha_{W}\right)$ is a submodule of $W$ for $(V, \mu, \alpha)$.

Define $\overline{\alpha_{W}}: W / \operatorname{Ker}\left(\alpha_{W}\right) \rightarrow \operatorname{Im}\left(\alpha_{W}\right)$ by $\overline{\alpha_{W}}(\bar{w})=\alpha_{W}(w)$. It is easy to verify that $\overline{\alpha_{W}}$ is an isomorphism. 
Corollary 5.8. If $\left(W, \alpha_{W}\right)$ is a irreducible bimodule of the simple multiplicative Hom-Jordan algebra $(V, \mu, \alpha)$ with $\alpha_{W}(a \cdot w)=\alpha(a) \cdot \alpha_{W}(w)$ for all $a \in V, w \in W$, then $\alpha_{W}$ is invertible.

Proposition 5.9. Suppose that $(V, \mu, \alpha)$ is a simple multiplicative Hom-Jordan algebra and $\left(W, \alpha_{W}\right)$ is a bimodule with $\alpha_{W}(a \cdot w)=\alpha(a) \cdot \alpha_{W}(w)$ for all $a \in V$, $w \in W$, and suppose that $\alpha_{W}$ is invertible. If $W$ is an irreducible module of the induced Jordan algebra $\left(V, \mu^{\prime}\right)$ with two compositions $w \cdot^{\prime} a=\alpha_{W}^{-1}(w \cdot a), a \cdot^{\prime} w=$ $\alpha_{W}^{-1}(a \cdot w)$ for all $a \in V, w \in W$, then $\left(W, \alpha_{W}\right)$ is an irreducible bimodule of $(V, \mu, \alpha)$.

Proof. Assume that $\left(W, \alpha_{W}\right)$ is reducible. Then there exists $W_{0} \neq\left\{0_{W}\right\}$ a subspace of $W$ such that $\left(W_{0},\left.\alpha_{W}\right|_{W 0}\right)$ is a submodule of $\left(W, \alpha_{W}\right)$. That is, $\alpha_{W}\left(W_{0}\right) \subseteq W_{0}$ and $a \cdot w \in W_{0}$, for any $a \in V, w \in W_{0}$. Hence, $a \cdot^{\prime} w=\alpha_{W}^{-1}(a \cdot w) \in \alpha_{W}^{-1}\left(W_{0}\right)=W_{0}$. So $W_{0}$ is a nontrivial submodule of $W$ for $\left(V, \mu^{\prime}\right)$, a contradiction. Hence, $\left(W, \alpha_{W}\right)$ is an irreducible bimodule of $(V, \mu, \alpha)$.

Remark 5.10. In [18], the author introduced another definition of Hom-Jordan algebras, using which one could verify that all the above results are also valid.

\section{ACKNOWLEDGEMENTS}

The authors would like to thank the referee for valuable comments and suggestions on this article.

\section{REFERENCES}

[1] B. Agrebaoui, K. Benali and A. Makhlouf, Representations of simple Hom-Lie algebras, $J$. Lie Theory 29 (2019), no. 4, 1119-1135. MR 4022147

[2] T. Ashihara, On a VOA associated with the simple Jordan algebra of type D, Comm. Algebra 39 (2011), no. 6, 2097-2113. MR 2813166

[3] S. Attan, Bimodules over Hom-Jordan and Hom-alternative algebras., preprint, 2018. arXiv:1804.00835 [math.RA].

[4] S. Benayadi and A. Makhlouf, Hom-Lie algebras with symmetric invariant nondegenerate bilinear forms, J. Geom. Phys. 76 (2014), 38-60. MR 3144357

[5] X. Chen and W. Han, Classification of multiplicative simple Hom-Lie algebras, J. Lie Theory 26 (2016), no. 3, 767-775. MR 3447949

[6] J. T. Hartwig, D. Larsson and S. D. Silvestrov, Deformations of Lie algebras using $\sigma-$ derivations, J. Algebra 295 (2006), no. 2, 314-361. MR 2194957

[7] N. Huang, L. Chen and Y. Wang, Hom-Jordan algebras and their $\alpha^{k}-(a, b, c)$-derivations, Comm. Algebra 46 (2018), no. 6, 2600-2614. MR 3778416

[8] N. Jacobson, General representation theory of Jordan algebras, Trans. Amer. Math. Soc. 70 (1951), 509-530. MR 0041118

[9] C. H. Lam, On VOA associated with special Jordan algebras, Comm. Algebra 27 (1999), no. 4, 1665-1681. MR 1679676

[10] D. Larsson and S. D. Silvestrov, Quasi-hom-Lie algebras, central extensions and 2-cocycle-like identities, J. Algebra 288 (2005), no. 2, 321-344. MR 2146132 
[11] D. Larsson and S. D. Silvestrov, Quasi-Lie algebras, in Noncommutative geometry and representation theory in mathematical physics, 241-248, Contemp. Math., 391, Amer. Math. Soc., Providence, RI, 2005. MR 2184027

[12] D. Larsson and S. D. Silvestrov, Quasi-deformations of $\mathfrak{s l}_{2}(\mathbb{F})$ using twisted derivations, Comm. Algebra 35 (2007), no. 12, 4303-4318. MR 2372334

[13] X. X. Li, Structures of multiplicative Hom-Lie algebras, Adv. Math. (China) 43 (2014), no. 6, 817-823. MR 3305521

[14] A. Makhlouf, Hom-alternative algebras and Hom-Jordan algebras, Int. Electron. J. Algebra 8 (2010), 177-190. MR 2660549.

[15] A. Makhlouf and S. D. Silvestrov, Hom-algebra structures, J. Gen. Lie Theory Appl. 2 (2008), no. 2, 51-64. MR 2399415

[16] Meng, D. J. Abstract algebras. II, Associative algebras., Beijing: Science Press, 2011 (in Chinese).

[17] Y. Sheng, Representations of hom-Lie algebras, Algebr. Represent. Theory 15 (2012), no. 6, 1081-1098. MR 2994017.

[18] D. Yau, Hom-Maltsev, Hom-alternative, and Hom-Jordan algebras, Int. Electron. J. Algebra 11 (2012), 177-217. MR 2876894.

[19] H. Zhao, Simplicities of VOAs associated to Jordan algebras of type $B$ and character formulas for simple quotients, J. Algebra 504 (2018), 364-390. MR 3784821

Chenrui Yao, Yao Ma, Liangyun Chen ${ }^{\bowtie}$

School of Mathematics and Statistics, Northeast Normal University, Changchun 130024, China yaocr827@nenu.edu.cn

may703@nenu. edu.cn

chenly640@nenu.edu.cn

Received: August 8, 2019

Accepted: March 3, 2020 